\title{
Desmoplastic trichoepithelioma: A clinicopathological study of three cases and a review of the literature
}

\author{
QIONGYU WANG ${ }^{1}$, DEEPAK GHIMIRE ${ }^{1}$, JUAN WANG $^{1}$, SUJU LUO $^{2}$, ZHENGXIAO LI $^{1}$, \\ HAO WANG $^{1}$, SONGMEI GENG ${ }^{1}$, SHENGXIANG XIAO ${ }^{1}$ and YAN ZHENG ${ }^{1}$ \\ ${ }^{1}$ Department of Dermatology, Second Affiliated Hospital of Xi'an Jiaotong University, Xi'an, Shaanxi; \\ ${ }^{2}$ Department of Dermatology, Tianjin Hospital of Tianjin Medical University, Tianjin, P.R. China
}

Received March 13, 2014; Accepted December 3, 2014

DOI: $10.3892 / \mathrm{ol} .2015 .3517$

\begin{abstract}
Desmoplastic trichoepithelioma (DTE) is a rare benign adnexal tumor with the characteristic features of asymptomatic, solitary, annular, indurated and centrally depressed papules or plaques, most commonly occurring in younger individuals on the face. Microscopically and clinically, DTE may be difficult to distinguish from other cutaneous adnexal neoplasms, particularly syringoma, cutaneous metastatic breast cancer, morpheaform basal cell carcinoma and microcystic adnexal carcinoma. The present study reports three cases of DTE. The first case was of a 45-year-old male with an asymptomatic flesh-colored plaque below the right edge of the outer canthus that had been present for seven years. The second case was of a 23-year-old female with an asymptomatic skin lesion on the right cheek that had slowly and progressively increased in size. The third case was of a 26 -year-old female who presented with a hard yellowish-white plaque, which gradually grew and formed a rectangular, $3 \times 4-\mathrm{cm}$ patch, on the tip of the left brow. This plaque was present for three years without evident cause or subjective symptoms. In all three cases, the routine systemic examinations and laboratory findings were normal. Histopathological and immunohistochemical findings from incisional biopsies of the lesions were consistent with a diagnosis of DTE. DTE treatment methods and immunohistochemical markers were analyzed by reviewing clinical pathological aspects in order to avoid a misdiagnosis and to provide the best available treatment approach for DTE.
\end{abstract}

\section{Introduction}

Trichoepitheliomas (TEs) are benign cutaneous neoplasms derived from the hair follicle. Three distinctive variants of TE

Correspondence to: Professor Yan Zheng, Department of Dermatology, Second Affiliated Hospital of Xi'an Jiaotong University, 157 Xiwu Road, Xi'an, Shaanxi 710004, P.R. China E-mail: zenyan66@126.com

Key words: desmoplastic trichoepithelioma, morpheaform basal cell carcinoma, microcystic adnexal carcinoma are recognized, namely, solitary TE, multiple TE and desmoplastic TE (DTE) (1). DTE is a rare benign adnexal tumor that is derived from basal cells in the outer root sheath of the hair follicle. The tumor occurs at an incidence of 1 in 5,000 skin biopsies in adults, and is usually observed in middle-aged females, but has been reported in all age groups and genders. DTE usually presents as an asymptomatic, flesh-colored, solitary, annular, indurated and centrally depressed papule or plaque $(2,3)$. The most commonly affected areas are the sun-exposed areas, particularly facial areas such as the cheeks, chin and forehead; less commonly, the tumors may be localized to the upper trunk area, the neck and the scalp (4).

The history of DTE goes back to 1904 when Hartzell described benign cystic epithelioma, which was clinically similar to DTE (5). In 1977, using a series of 49 cases, Brownstein and Shapiro (6) described the microscopic features of DTE, and histologically noted narrow strands of basaloid tumor cells, keratinous cysts and a desmoplastic stroma. Since then, these features have remained as a unique triad for the dermatopathology of DTE.

DTE lesions are usually superficial and rarely reach the lower dermis. The tendency for perineural and intraneural invasion, such as has been found in other cutaneous malignancies, has rarely been described in the previous literature. According to these studies, there is no pleomorphism, mitotic figures or apoptotic activity in the epithelium, which morphologically resembles the main tumor. Therefore, it is clear that DTE should also be listed with other cutaneous neoplasms showing perineural involvement, particularly the desmoplastic malignancies, and that care should be employed in following any aggressive treatment approach, much like for other cutaneous malignancies, particularly when lesions are located in cosmetically sensitive areas. Immunohistochemical studies reveal the consistent expression of cytokeratin (CK)20 (7) for Merkel cells surrounded by stromal cells.

Clinical features and histopathological features may aid in the diagnosis of DTE (8). The most common features of DTE are that the tumor is slow-growing, white-gray to flesh-colored, indurated, centrally depressed, non-ulcerated and 2-18 $\mathrm{mm}$ in diameter (9). The tumor has an annular border, is present as a papule or plaque, and predominately occurs on the face. Diagnosis based only on the clinical background may be difficult is certain cases, and for those cases, biopsy may be of use. The 
lesion is usually well circumscribed, symmetrical and confined to the papillary dermis and upper two-thirds of the reticular dermis. The presence of narrow strands of epithelial basaloid cells, numerous horn cysts, a dense fibrous stroma, a foreign body-type granulomatous reaction, calcification and osteoma may serve as diagnostic characteristics (10).

Clinically, DTE is difficult to distinguish from other skin lesions caused by certain diseases, such as breast cancer. In this study, three cases of DTE were presented and the associated literature was reviewed. The aim of the study was to improve understanding with regard to the clinical diagnosis and treatment of DTE. Written consent was obtained from each patient or their relatives for publication of this study.

\section{Case reports}

Case one. In January 2009, a 45-year-old male presented to the Second Affiliated Hospital of Xi'an Jiatong University (Xi'an, China) with an asymptomatic flesh-colored plaque below the right edge of the outer canthus that had been apparent for seven years. The lesion had first become apparent at 38 years old, when the patient was injured by a piece of iron that left a small scar below the right edge of the outer canthus. There was no pain, itching or any associated symptoms, so no further management was provided. However, in the last seven years, the lesion slowly and progressively increased in size without any symptoms or known cause.

Dermatological examination of the lesion revealed it to be flesh-colored and centrally depressed, with elevated borders of $\sim 15 \mathrm{~mm}$ in size, and located laterally over the right periorbital region. The lesion was not ulcerated (Fig. 1). The patient's eating habits, bowel habits and urination were normal. There was no family history of similar disease and no associated symptoms. Upon histological examination, nests of small strands and cords of epithelial elements were observed within the upper and mid dermis. The chords and nests of basaloid cells varied in size and were embedded in a dense stroma. These aggregations were rimmed by bundles of collagen fibers. Multiple horn cysts were also apparent in the stroma and were lined by stratified squamous epithelium. A thin drag line elicited by focal calcification was also observed. Mitotic figures were not apparent. Pleomorphism and peripheral palisading were not observed. By reviewing the histopathological findings and correlating these with the clinical findings, a diagnosis of DTE was established (Fig. 2). The immunohistochemistry revealed CK20-positive cells (Fig. 3A) diffusely scattered within strands of the tumor, including the wall of a horn cyst, and cluster of differentiation (CD)34-positive cells (Fig. 3B) surrounding the tumor mass. Immunohistochemistry for epithelial membrane antigen (EMA; Fig. 3C) and androgen receptor (AR; Fig. 3D) expression was negative. The patient underwent surgery with complete resectioning with no recurrence.

Case two. In October 2010, a 23-year-old female presented to the Second Affiliated Hospital of Xi'an Jiatong University with an asymptomatic skin lesion that had been apparent for nine years and was slowly and progressively increasing in size. There was no relevant medical history of any disease and no family history of a similar case. Examination of the lesion revealed a small, flesh-colored, centrally depressed, bean-sized single

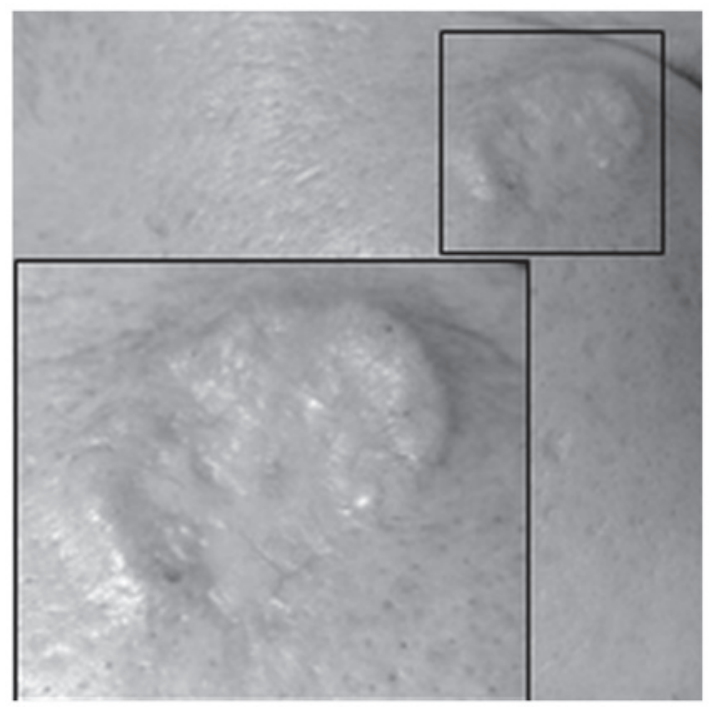

Figure 1. Case one: Microscopic appearance of the flesh-colored and centrally depressed lesion, with elevated borders, which was located laterally over the right periorbital region.

lesion located on the right cheek, with raised borders (Fig. 4). A systemic examination was performed, but did not reveal any regional lymphadenopathy or cutaneous abnormalities. The patient's eating habits, bowel habits and urination were normal. A biopsy specimen obtained from the site revealed small strands of basaloid cells in a desmoplastic stroma, with keratinaceous cysts positioned adjacently and attached to the basaloid cells. Following a review of the clinicohistopathological findings, a diagnosis of DTE was established (Fig. 5).

The immunohistochemistry revealed the presence of a few CK20-positive cells (Fig. 6A) scattered within the strands of the tumor. CD-34-positive cells (Fig. 6B) surrounded the tumor mass. Immunohistochemistry for EMA (Fig. 6C) and AR (Fig. 6D) was negative, while B-cell lymphoma (Bcl)-2 expression (Fig. 6E) was weakly positive in the basal layer. The patient underwent surgery with complete resectioning with no recurrence.

Case three. In November 2012, a 26-year-old female presented to the Second Affiliated Hospital of Xi'an Jiatong University with a hard yellowish-white plaque, which had gradually grown over three years and formed a rectangular, 3x4-cm patch (Fig. 7) on the tip of the left brow, without evident cause or subjective symptoms. There was no relevant medical history of any disease and no family history of a similar case. Systemic and laboratory examinations revealed no abnormalities. Pathological examination was performed; the hematoxylin and eosin staining of the biopsy specimen is shown in Fig. 8, which revealed mild atrophy of the epidermis, with large cords identified in the shallow and middle dermis. In addition, hyperplasia of the connective tissue was observed at the horn cyst. The patient underwent surgery with complete resectioning with no recurrence.

\section{Discussion}

DTE is a relatively rare, benign, cutaneous neoplasm, whose microscopical and histological features were first described 

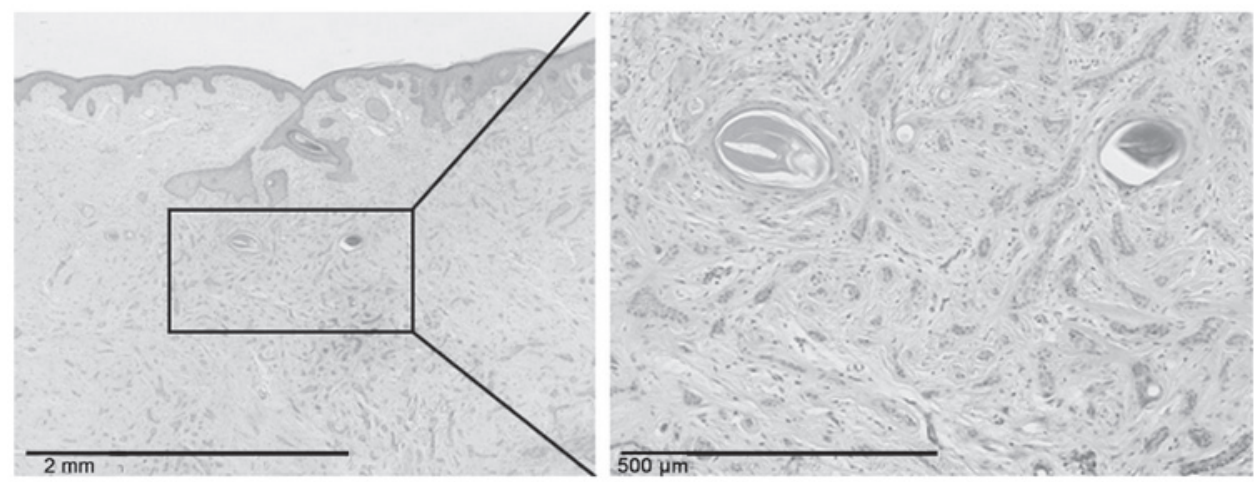

Figure 2. Case one: Light microscopy biopsy revealed no clear abnormalties of the epidermis and the presence of a mass branching tumor cells at the shallow and middle dermis, as well as the proliferation of collagen (hematoxylin and eosin stain).
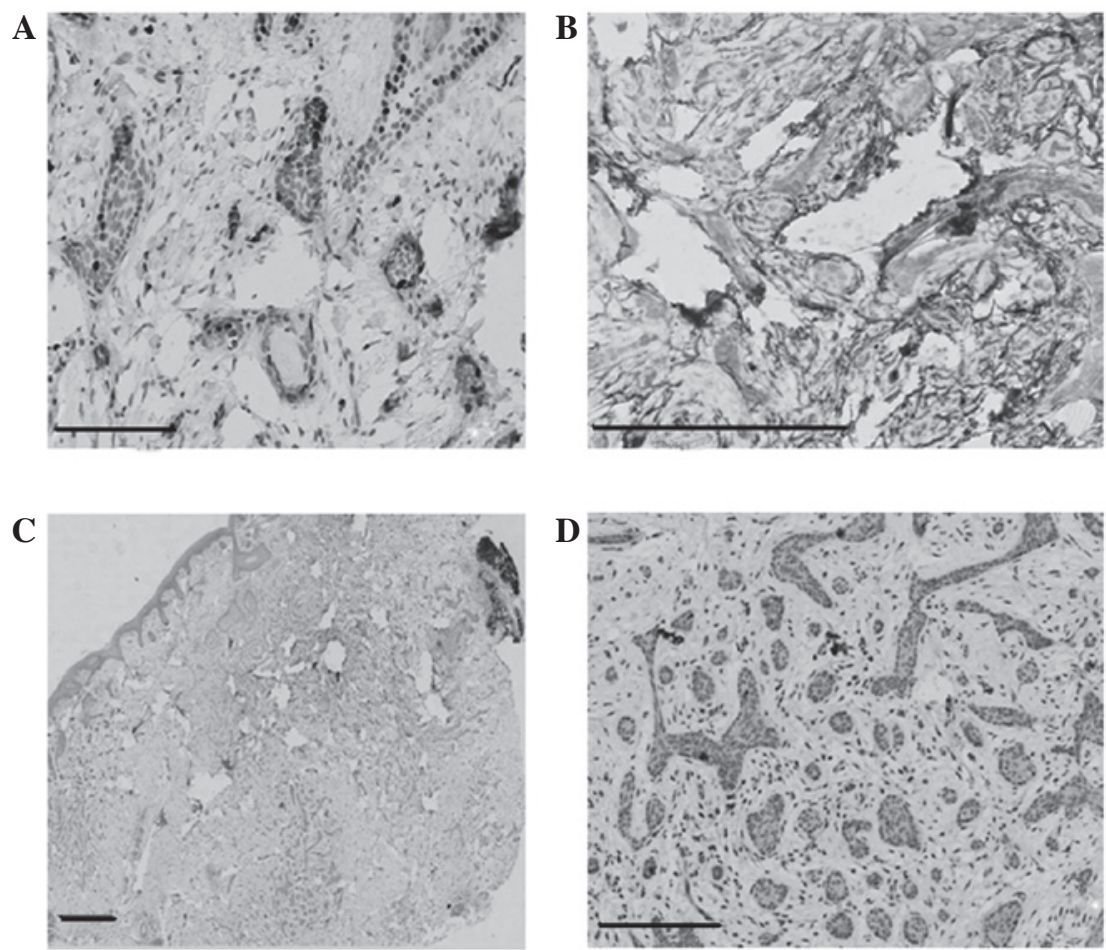

Figure 3. Case one: Immunohistochemical staining showing (A) diffusely scattered cytokeratin 20-positive cells within strands of the tumor and (B) cluster of differentiation 34-positive cells surrounding the tumor mass; and negative (C) epithelial membrane antigen and (D) androgen receptor expression. Scale bars, $100 \mu \mathrm{m}$.

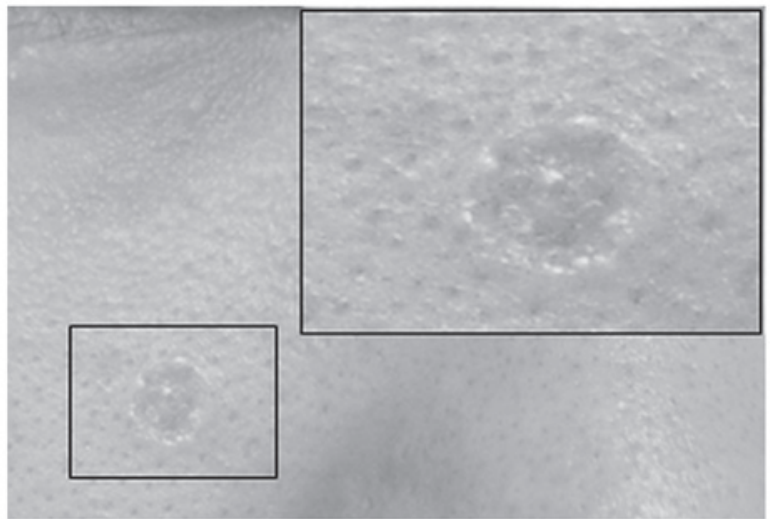

Figure 4. Case two: Microscopic appearance of the small, flesh-colored, centrally depressed, bean-sized single lesion, with raised borders, which was located on the right cheek. by Brownstein and Shapiro in 1977 (6). The lesion has been reported in previous studies throughout the literature as several different entities, including solitary TE, epithelioma adenoides cysticum, morphea-like epithelioma and sclerosing epithelial hamartoma $(11,12)$. DTE usually presents as a slow-growing, asymptomatic, solitary, indurated plaque or papule. The lesion has a raised annular border and depressed non-ulcerating center. DTE most commonly occurs on sun-exposed areas, particularly the face, and is often mistaken for a basal cell carcinoma (BCC) in older patients. Moreover, DTE is believed to occur more on the right side of the face, with the most common site being the cheek, followed by the nose, chin, forehead, periorbital region and lips $(2,6)$. Females are more commonly affected than males, with occurrences in the age range of 8-81 years (13). 

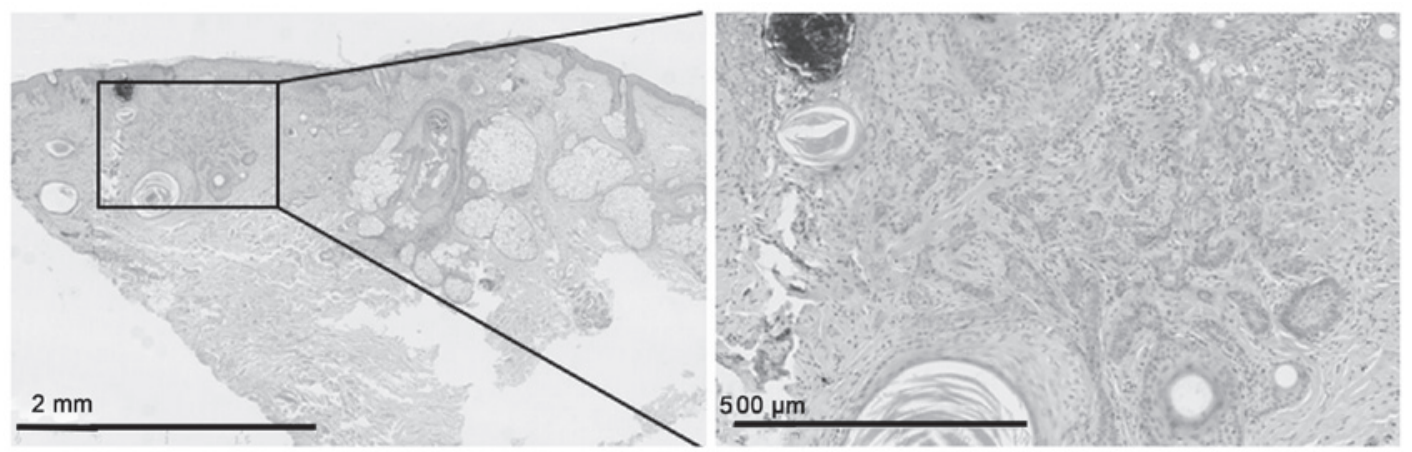

Figure 5. Case two: Light microscopy biopsy revealed thickening of the epidermis and the presence of a mass of branching tumor cells at the shallow and middle dermis, as well as the proliferation of collagen (hematoxylin and esoin stain).

A

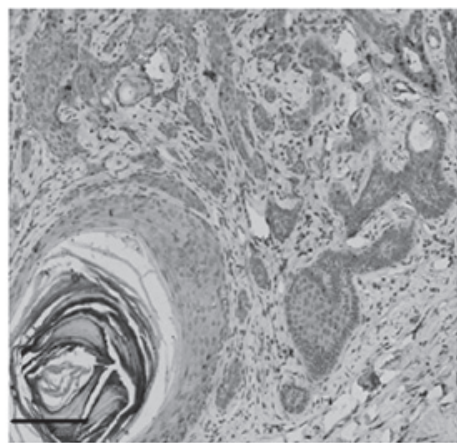

B

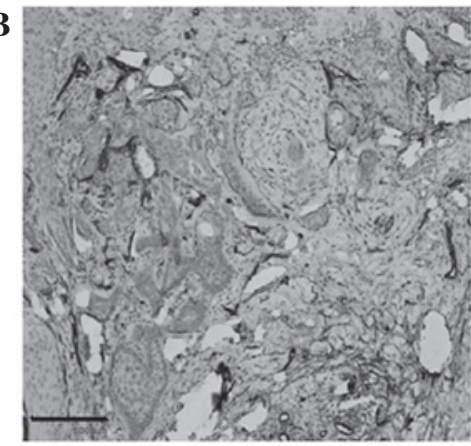

C

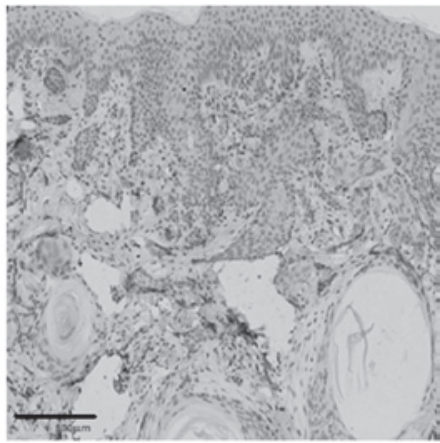

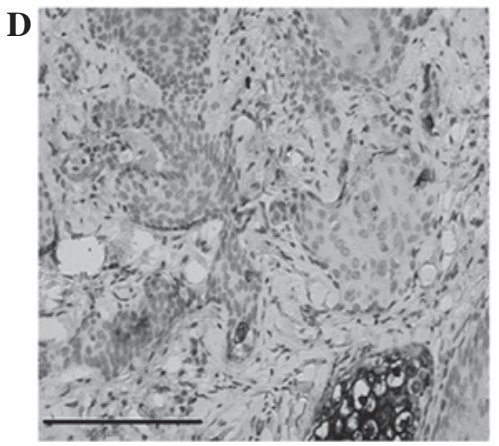

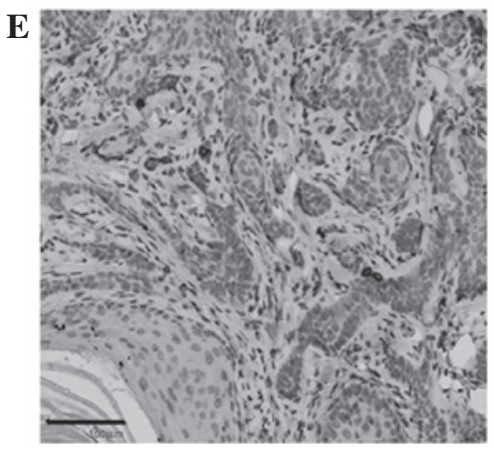

Figure 6. Case two: Immunohistochemical staining revealing (A) a few cytokeratin 20-positive cells within the strands of the tumor and (B) cluster of differentiation 34-positive cells surrounding the tumor mass; negative (C) epithelial membrane antigen and (D) androgen receptor expression; and (E) weakly positive B-cell lymphoma 2 expression. Scale bars, $100 \mu \mathrm{m}$.

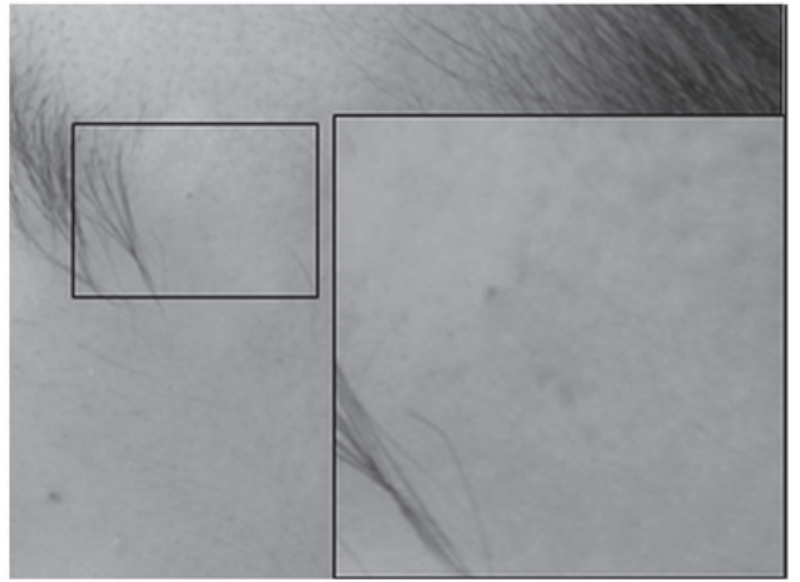

Figure 7. Case three: Microscopic appearance of the hard yellowish-white plaque, which had formed a rectangular, $3 \times 4-\mathrm{cm}$ patch, and was located on the tip of the left brow.
On histopathological examination, the lesion is usually well circumscribed, symmetrical and confined to the papillary dermis and upper two-thirds of the reticular dermis (9). The three cases examined within the present study exhibited the typical clinical and histopathological features of DTE that have been described by previous studies $(4,5,10)$. The depressed, non-ulcerating, and raised angular border that is described in previous studies $(4,5,10)$ was a typical feature in the present cases. The triad of histopathological characteristics first described by Brownstein and Shapiro (6), i.e., narrow strands of basaloid tumor cells, keratinous cysts and a desmoplastic stroma, was a consistent presence in all three of the current cases. Another feature of DTE is the presence of horn cysts and frequent calcification. In the present study, multiple horn cysts and focal calcifications were observed on all cases. There were no signs of pleomorphism, mitotic figures or apoptotic activity in the epithelium. Immunological 


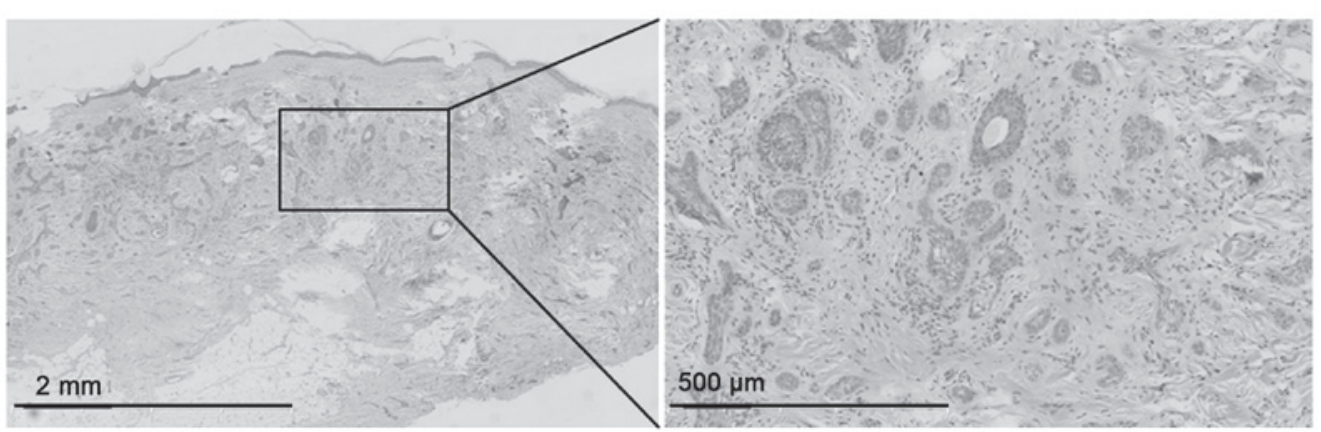

Figure 8. Case three: Light microscopy biopsy revealed mild atrophy of the epidermis and the presence of a mass of branching tumor cells at the shallow and middle dermis,a s well as the proliferation of collagen (hematoxylin and eosin stain).

markers were studied in two cases. Each of the cases showed CK20 expression, however, in case one, numerous cells expressed strong CK20 expression compared with a few cells expressing CK20 and multiple cells weakly expressing CK20 in case two. Bcl-2 was also mainly expressed in the basal layers. EMA expression was negative, while CD34 expression was positive around the tumor mass.

The diagnosis of DTE may occasionally be difficult, even when assessed by an expert, and particularly when the tumor mimics other benign and malignant tumors. DTE may clinically and histopathologically mimic syringoma, morpheaform BCC (MBCC), microcystic adnexal carcinoma (MAC), conventional TE and other tumors. While histology findings combined with clinical features may be useful in making a definitive diagnosis of some of these lesions $(14,15)$, MBCC may still be misdiagnosed. Other frequently associated conditions that may mimic similar clinical and histological features are sebaceous hyperplasia, granuloma annulare, scar tissue and cutaneous squamous cell carcinoma (16). The features of DTE, as aforementioned, may be extremely hard to distinguish when provided with a small sample of biopsy specimen. Also, as the majority of the biopsy specimens may be superficial, it may be hard to make a full pathological evaluation. Certain studies have suggested that the thin-walled epidermis and the lack of surface telangiectasias, along with the aforementioned features, can aid in the diagnosis of DTE $(2,10)$. However, differentiating DTE from BCC and MAC remains challenging. Takei et al (17) discovered that the majority of clinically diagnosed DTE cases were actually BCC. Other lesions in their series were sebaceous hyperplasias, hamartoma, TE, melanocystic nevi and keratoses.

Several attempts have been made to overcome those diagnostic challenges in ultrastructural pathology (14), molecular pathology (18), immunofluorescence (19) and immunohistochemical $(17,20-23)$ studies. Immunohistochemical markers that have been proposed as criteria for the diagnosis and differentiation between DTE and MBCC are CK20 (for Merkel cells), p53, p75, CD10, CD34, PHLDA, AR, Ki-67 and Bcl-2. Costache et al (18) investigated several immunohistological markers, namely CK20, AR, Ki-67, CD34, p53, Bcl-2 and CD10. The study attempted to re-evaluate the histomorphological and immunohistochemical criteria previously proposed by Takei et al (17), which were available for the differentiation of DTE from MBCC, in order to figure out which of the criteria are the most reliable for a definitive diagnosis. The study found that CK20 and AR are the most reliable immunohistochemical markers for the differentiation between DTE and MBCC, supporting the data of previous studies by Abesamis-Cubillan et al (22) and Izikson et al (23). However, the study also suggested that the number of Merkel cells in DTE may vary from a large number to very few or even one. Therefore, relying on CK20 for small biopsy specimens may remain problematic, particularly in view of the ever decreasing size of skin biopsies and the increased use of the shave biopsy technique, which often yields only superficial small specimens. Costache et al (20) further concluded that $\mathrm{Ki}-67$ and Bcl-2 were not useful markers in the differentiation between DTE and MBCC.

Although DTE may be diagnosed using the clinical and morphological features alone, in certain cases, this may be challenging for a dermatologist. DTE may resemble other benign and malignant tumors clinically and histologically, including MBCC, MAC, cutaneous metastatic breast cancer and syringoma $(9,24)$.

Syringoma is a benign, adnexal neoplasm with ductal differentiation. The condition usually presents as small, multiple, skin-colored papules over the cheeks and lower eyelids. The lesions are usually asymptomatic and tend to first appear at puberty. The histopathological features of syringoma show multiple eccrine ducts, which are lined by two layers of cuboidal epithelium and are scattered within a fibrous stroma in the dermis. The main histopathological differential diagnosis includes MBCC, MAC, eccrine syringocarcinoma and DTE. Syringoma, unlike DTE, is often observed as multiple lesions on the periorbital region. The lesions are generally confined to the superficial dermis and consist of tubular structures (2). All these features are absent in DTE. Narrow strands of basaloid cells, foreign body granulomas and calcification may rarely be observed in syringoma, but may frequently be found in DTE (25). DTEs also tend to be solitary and lack the ductal differentiation observed in syringomas. Additionally, the presence of horn cysts, calcification, follicular differentiation and long epithelial strands can distinguish DTE from syringoma, where these features are not commonly observed. Moreover, immunohistochemical markers may also aid in differentiating between DTE and syringoma. The immunohistochemical CK20 marker for Merkel cells is nearly always immunopositive for DTE, but rarely or never for syringoma (7). DTE is also negative 
Table I. Major features for differentiating between DTE and syringoma.

\begin{tabular}{lcc}
\hline Features & DTE & Syringoma \\
\hline Narrow strands of tumor cells & Constant & Unusual \\
Hard, annular lesions & Typical & Rare \\
Horn cysts & Constant & Rare \\
Solitary tumors & Common & Rare \\
Epidermal hyperplasia & Common & Rare \\
Ductal differentiation & Rare & Common \\
Foreign body granuloma & Frequent & Rare \\
Calcification & Frequent & Rare \\
Periorbital involvement & Rare & Common \\
Immunohistochemical markers & & Rarely positive \\
CK20 & Strong positive & Positive \\
Carcinoembryonic antigen & Negative & \\
\hline
\end{tabular}

DTE, desmoplastic trichoepithelioma; CK, cytokeratin.

Table II. Major features for differentiating between DTE and cutaneous metastastic breast cancer.

\begin{tabular}{|c|c|c|}
\hline Features & DTE & $\begin{array}{c}\text { Cutaneous metastatic } \\
\text { breast cancer }\end{array}$ \\
\hline Chest involvement & No & Common \\
\hline Annular lesions & Typical & No \\
\hline Horn cysts & Common & Rare \\
\hline Narrow strands of basaloid cells & Constant & Rare \\
\hline Large masses of tumor cells & Never & Common \\
\hline Epidermal hyperplasia & Common & Rare \\
\hline Keratin granulomas and calcification & Common & Rare \\
\hline Cellular atypia & Never & Common \\
\hline
\end{tabular}

DTE, desmoplastic trichoepithelioma.

for carcinoembryonic antigen $(26,27)$ compared with syringoma, which shows a positive reaction in the luminal cells. The most common features that may aid in differentiating DTE from syringoma are listed in Table I.

In the majority of cases, cutaneous metastasis occurs following the initial diagnosis of the primary cancer. In the minority of cases, metastasis may be discovered at the same time or prior to the diagnosis of carcinoma. Breast cancer is one of the most common malignancies to spread to the skin $(28,29)$. It is estimated that $30 \%$ of breast cancers have the tendency to metastasize. Although the most common sites of cutaneous metastatic breast cancer are the chest and abdomen, metastasis can less commonly be discovered on the scalp, face, neck, upper extremities, abdomen and back. Patients may present with rapidly-growing, asymptomatic, firm, scar-like nodules or tumors on the face, which may mimic DTE (30). Any rapidly-growing lesions should warrant careful consideration of the possibility of metastasis. The most common features that may aid in differentiating between DTE and cutaneous metastatic breast cancer are listed in Table II.

$\mathrm{MBCC}$, also known as sclerosing $\mathrm{BCC}$ is a rare $\mathrm{BCC}$ variant that exhibits aggressive characteristics and an atypical clinical presentation. The preponderance of BCCs are nodular or superficial. MBCC is considered a potentially more aggressive subtype necessitating complete surgical excision, as opposed to the benign nature of DTE (31).

MBCC presents as solitary, yellowish or skin-colored, pale, firm, ill-defined, waxy or scar-like, flat or slightly depressed lesions, which resemble numerous other benign lesions, such as DTE. The head and neck regions, particularly the face and less so the trunk and limbs, are the most frequently affected by MBCC (32).

MBCC may clinically and histologically mimic DTE. Since the two tumors are each composed of follicular germinative cells, numerous morphological characteristics are common between them. There is significant overlap between DTE and MBCC (21). Differentiating between these two 
Table III. Features for differentiating between DTE and MBCC.

\begin{tabular}{|c|c|c|}
\hline Features & DTE & $\mathrm{MBCC}$ \\
\hline Symmetry & Often symmetrical & Often asymmetrical \\
\hline Annular lesion & Characteristics & Rare \\
\hline Horn cyst & Always present & Very rare \\
\hline Ulceration & Rare & Common \\
\hline Depression in the center & Common & Uncommon \\
\hline presense of larger aggregations & Uncommon & Common \\
\hline Rims of collagen bundles & Constant & Less common \\
\hline Small strands of epithelial elements & Frequent & Less frequent \\
\hline Calcification & Common & Uncommon \\
\hline Follicular, sebaceous, infundibular differentiation & Common & Uncommon \\
\hline Clefts between aggregations and stroma & Rare & Often \\
\hline Mitotic figures & Rare & Frequent \\
\hline Cut artefacts & Common & Uncommon \\
\hline Granulomatous inflammation & Frequently observed & Infrequently seen \\
\hline Solar elastosis below the lesion & Rare & Common \\
\hline \multicolumn{3}{|l|}{ Immunohistochemical markers } \\
\hline CK20 & Strongly positive & Negative \\
\hline AR & Rare & Common \\
\hline
\end{tabular}

DTE, desmoplastic trichoepithelioma; MBCC, morpheaform basal cell carcinoma; CK, cytokeratin; AR, androgen receptor.

Table IV. Features for differentiating between DTE and MAC.

\begin{tabular}{|c|c|c|}
\hline Features & DTE & MAC \\
\hline Symmetry & Symmetrical & Asymmetrical \\
\hline Ductal structures & Infrequent & Frequent \\
\hline $\begin{array}{l}\text { Intramuscular, perichondral } \\
\text { and perineural involvement }\end{array}$ & Uncommon & Common \\
\hline Circumscribe & Well circumscribed & Poorly circumscribed \\
\hline Infilteration & $\begin{array}{l}\text { Confined to the papillary dermis and the } \\
\text { upper two-thirds of the reticular dermis }\end{array}$ & $\begin{array}{l}\text { Extending beyond the } \\
\text { reticular dermis }\end{array}$ \\
\hline
\end{tabular}

DTE, desmoplastic trichoepithelioma; MAC, microcystic adnexal carcinoma.

neoplasms may be challenging for anyone. Like DTE, MBCC also consists of infiltrating strands and islands of basaloid and monomorphic cells embedded within a dense fibrous and sclerotic stroma.

DTE and MBCC may be differentiated between using five distinct clinicohistopathological findings (21): Annular lesions, horn-cysts, epidermal hyperplasia, Keratin granulomas and calcification. The large masses of tumor cells that are often encountered in MBCC are rarely found in DTE (21). A number of the common features that may aid in differentiating between DTE and MBCC are listed in Table III.

MAC is a rare adnexal neoplasm that normally occurs on the head and neck region, particularly the central face (33). MAC clinically presents as a slow-growing, firm, flesh-colored and indurated plaque or nodule, with diffuse, ill-defined margins, occasionally with overlying telangiectasia. MAC is large, poorly circumscribed and asymmetric, and extends into the subcutaneous fat. The neoplasm consists predominantly of proliferating tubular structures (34).

While DTE is a benign neoplasm with indolent behavior, MAC can be highly aggressive, resulting in substantial local destruction and possible metastasis. Although MAC has widely been recognized as a discrete clinicopathological entity, confusion with other benign adnexal tumors, particularly DTE, remains likely (18). Superficial biopsies result in the misdiagnosis of MAC as squamous cell carcinoma, syringoma or DTE in up to $30 \%$ of cases. A number of the features (21) that may aid in differentiating between DTE and MAC are listed in Table IV. 
Immunohistochemical markers have also been studied to aid in differentiating between DTE and MAC. These markers include CK20, CK7, CK15, CD10 and BerEP4 (20). However, none of the proposed immunohistochemical markers are believed to be totally reliable. Debate remains over the reliability of the markers $(23,35)$. As a consequence, further studies are required in order to find a definitive diagnostic marker able to differentiate between MAC and DTE.

Several approaches, including laser surgery, dermabrasion, topical 5\% imiquimod (14), curettage and electrodesiccation, and radiosurgical ablation, have been attempted with some success, but the chances of recurrence for these techniques may be higher than that for local surgical excision $(3,14,36)$. It is also important to consider that the majority of biopsy specimens obtained during these methods may only be superficial, resulting in a poor pathological evaluation. Moreover, these techniques may not permit histological margin analysis and may not be appropriate for high-risk tumors resembling DTE. Local surgical excision is the treatment of choice for DTE and is considered as a first-line treatment for the majority of benign tumors. Although complete remission with minimal recurrence can be achieved with this technique, post-surgical complications, including scarring and hypopigmentation, remain the main problem, particularly for the cosmetically sensitive areas such as the face, where minimizing the occurrence of any complications is extremely important. The study by Mamelak et al (2) recommends Mohs micrographic surgery for treating DTE, in order to prevent recurrence and local invasion. A review of the literature shows that certain studies agree on the fact that aggressiveness and local invasion for DTE is extremely rare $(37,38)$. Moreover, Mohs micrographic surgery is relatively expensive compared with other alternative or surgical modalities (39). For classical cases with definitive benign results, DTE can be cost-effectively managed by closed monitoring only, with regular follow-ups or local excision if required. However, in cases with atypical clinical and histological features and where there is concern about the tumor arising in cosmetically sensitive areas like the face, in which the sparing of normal surrounding tissue is important, Mohs surgery may be beneficial (38).

From the results of the present study and the previous literature, it may be concluded that DTE is a particularly rare benign adnexal tumor. The treatment of choice is local excision, but a 'wait and watch' policy can be used as a management technique in those cases where the clinical features are typical to DTE. For a tumor as rare as DTE, the data for recurrence is not reliable; therefore, the specific recurrence rate cannot be reliably calculated. The tumor has been shown to share a number of clinicohistopathological similarities with MBCC and MAC. Although histopathological and immunohistochemical markers may aid in the differentiation of other malignant tumors, specific diagnostic techniques for the differentiation of this tumor are still lacking. While the majority of cases may be left untreated, the diagnosis and differentiation of DTE remains essential, as the treatment and prognosis of other tumors mimicking DTE is different. Overall, the low incidence of DTE limits the histopathological and immunohistochemical observations, and the treatment studies that may be performed.

\section{References}

1. D'Souza M, Garg BR, Ratnakar C and Agrawal K: Multiple trichoepitheliomas with rare features. J Dermatol 21: 582-585, 1994.

2. Mamelak AJ, Goldberg LH, Katz TM, Graves JJ, Arnon O and Kimyai-Asadi A: Desmoplastic trichoepithelioma. J Am Acad Dermatol 62: 102-106, 2010.

3. Moynihan GD, Skrokov RA, Huh J, Pardes JB and Septon R: Desmoplastic trichoepithelioma. J Am Acad Dermatol 64: 438-439, 2011

4. Brichta RF and Feldman BD: Multiple flesh-colored facial papules. Multiple trichoepitheliomas. Arch Dermatol 126: 953, 956, 1990.

5. Lazorik FC and Wood MG: Multiple desmoplastic trichoepitheliomas. Arch Dermatol 118: 361-362, 1982.

6. Brownstein MH and Shapiro L: Desmoplastic trichoepithelioma. Cancer 40: 2979-2986, 1977.

7. Katona TM, Perkins SM and Billings SD: Does the panel of cytokeratin 20 and androgen receptor antibodies differentiate desmoplastic trichoepithelioma from morpheaform/infiltrative basal cell carcinoma? J Cutan Pathol 35: 174-179, 2008.

8. López-Navarro N, Alcaide A, Gallego E, Herrera-Acosta E, Castillo R and Herrera E: Dermatoscopy in the diagnosis of combined desmoplastic trichoepithelioma and naevus. Clin Exp Dermatol 34: e395-e396, 2009.

9. Khelifa E, Masouyé I, Kaya G and Le Gal FA: Dermoscopy of desmoplastic trichoepithelioma reveals other criteria to distinguish it from basal cell carcinoma. Dermatology 226: 101-104, 2013.

10. Ardigo M, Zieff J, Scope A, et al: Dermoscopic and reflectance confocal microscope findings of trichoepithelioma. Dermatology 215: 354-358, 2007.

11. Steigleder GK: Solitary trichoepithelioma. Hautarzt 23: 323-324, 1972 (In German).

12. Verma KC and Chaudhry SD: Epithelioma adenoides cysticum (trichoepithelioma). Indian J Med Sci 27: 627-629, 1973.

13. Abbas O, Richards JE and Mahalingam M: Fibroblast-activation protein: a single marker that confidently differentiates morpheaform/infiltrative basal cell carcinoma from desmoplastic trichoepithelioma. Mod Pathol 23: 1535-1543, 2010.

14. Yamamoto O, Hamada T, Doi Y, Sasaguri Y and Hashimoto H: Immunohistochemical and ultrastructural observations of desmoplastic trichoepithelioma with a special reference to a morphological comparison with normal apocrine acrosyringeum. J Cutan Pathol 29: 15-26, 2002.

15. Mitcov M, Scrivener Y and Cribier B: Desmoplastic trichoepithelioma: a clinicopathological study, including a comparison with morpheiform basal cell carcinoma. Ann Dermatol Venereol 136: 501-507, 2009 (In French).

16. Sumithra S, Jayaraman M and Yesudian P: Desmoplastic trichoepithelioma and multiple epidermal cysts. Int J Dermatol 32: 747-748, 1993.

17. Takei Y, Fukushiro S and Ackerman AB: Criteria for histologic differentiation of desmoplastic trichoepithelioma (sclerosing epithelial hamartoma) from morphea-like basal-cell carcinoma. Am J Dermatopathol 7: 207-221, 1985.

18. Tse JY, Nguyen AT, Le LP and Hoang MP: Microcystic adnexal carcinoma versus desmoplastic trichoepithelioma: a comparative study. Am J Dermatopathol 35: 50-55, 2013.

19. Mizutani Y, Iwamoto I, Kanoh H, Seishima M and Nagata K: Expression of drebrin, an actin binding protein, in basal cell carcinoma, trichoblastoma and trichoepithelioma. Histol Histopathol 29: 757-766, 2014.

20. Costache M, Bresch M and Böer A: Desmoplastic trichoepithelioma versus morphoeic basal cell carcinoma: a critical reappraisal of histomorphological and immunohistochemical criteria for differentiation. Histopathology 52: 865-876, 2008.

21. Sellheyer K, Nelson P, Kutzner H and Patel RM: The immunohistochemical differential diagnosis of microcystic adnexal carcinoma, desmoplastic trichoepithelioma and morpheaform basal cell carcinoma using BerEP4 and stem cell markers. J Cutan Pathol 40: 363-370, 2013.

22. Abesamis-Cubillan E, El-Shabrawi-Caelen L and LeBoit PE: Merked cells and sclerosing epithelial neoplasms. Am J Dermatopathol 22: 311-315, 2000.

23. Izikson L, Bhan A and Zembowicz A: Androgen receptor expression helps to differentiate basal cell carcinoma from benign trichoblastic tumors. Am J Dermatopathol 27: 91-95, 2005. 
24. Vorechovský I, Undén AB, Sandstedt B, Toftgård R and Ståhle-Bäckdahl M: Trichoepitheliomas contain somatic mutations in the overexpressed PTCH gene: support for a gatekeeper mechanism in skin tumorigenesis. Cancer Res 57: 4677-4681, 1997.

25. Ahmed M: Cutaneous metastases from breast carcinoma. BMJ Case Rep 2011: bcr0620114398, 2011.

26. Wick MR, Cooper PH, Swanson PE, Kaye VN and Sun TT: Microcystic adnexal carcinoma. An immunohistochemical comparison with other cutaneous appendage tumors. Arch Dermatol 126: 189-194, 1990.

27. Hoang MP, Dresser KA, Kapur P, High WA and Mahalingam M: Microcystic adnexal carcinoma: an immunohistochemical reappraisal. Mod Pathol 21: 178-185, 2008.

28. Prabhu S, Pai SB, Handattu S, Kudur MH and Vasanth V: Cutaneous metastases from carcinoma breast: the common and the rare. Indian J Dermatol Venereol Leprol 75: 499-502, 2009.

29. Moore S: Cutaneous metastatic breast cancer. Clin J Oncol Nurs 6: 255-260, 2002

30. Lai YL, Chang HH, Huang MJ, et al: Combined effect of topical arsenic trioxide and radiation therapy on skin-infiltrating lesions of breast cancer-a pilot study. Anticancer Drugs 14: 825-828, 2003.

31. Richman T and Penneys NS: Analysis of morpheaform basal cell carcinoma. J Cutan Pathol 15: 359-362, 1988

32. Bozikov K and Taggart I: Metastatic basal cell carcinoma: is infiltrative/morpheaform subtype a risk factor? Eur J Dermatol 16 691-692, 2006.

33. Pugh TJ, Lee NY, Pacheco T and Raben D: Microcystic adnexal carcinoma of the face treated with radiation therapy: a case report and review of the literature. Head Neck 34: 1045-1050, 2012.
34. Fischer S, Breuninger H, Metzler $\mathrm{G}$ and Hoffmann J: Microcystic adnexal carcinoma: an often misdiagnosed, locally aggressive growing skin tumor. J Craniofac Surg 16: 53-58, 2005 .

35. Merritt BG, Snow SN and Longley BJ: Desmoplastic trichoepithelioma, infiltrative/morpheaform BCC, and microcystic adnexal carcinoma: differentiation by immunohistochemistry and determining the need for Mohs micrographic surgery. Cutis 85: 254-258, 2010.

36. Jedrych J and McNiff JM: Expression of p75 neurotrophin receptor in desmoplastic trichoepithelioma, infiltrative basal cell carcinoma, and microcystic adnexal carcinoma. Am J DermatoPathol 35: 308-315, 2013

37. Karikal A, Shetty P, Karikal A and Shetty SR: Multiple trichoepitheliomas: A rare occurrence. South Asian J Cancer 2: 54, 2013.

38. Yiltok SJ, Echejoh GO, Mohammad AM, Ituen AM, Igoche MI and Dades OT: Multiple familial trichoepithelioma: a case report and review of literature. Niger J Clin Pract 13: 230-232, 2010.

39. Clayton AS and Stasko T: Treatment of nonmelanoma skin cancer in organ transplant recipients: review of responses to a survey. J Am Acad Dermatol 49: 413-416, 2003. 